\title{
Sentido, gravedad y razones del ciberplagio entre el alumnado de ESO de Andalucía
}

\section{Cyber-Plagiarims in the secondary andalusian students: sense, gravity and reasons}

\author{
Mónica López-Gil \\ Doctora en Educación, PDI \\ (Universidad de Cádiz) \\ Félix Angulo Rasco \\ Investigador \\ (Centro de Investigación para la Educación Inclusiva CIE 16009) \\ Miembro del Claustro de Magister Escuela de Pedagogía \\ (Universidad Católica de Valparaíso) \\ Rosa Vázquez-Recio \\ Profesora Titular del Departamento de Didáctica \\ (Universidad de Cádiz)
}

Fecha de recepción: 12 de enero de 2017

Fecha de revisión: 27 de junio de 2017

Fecha de publicación: 1 de julio de 2017

Para citar este artículo: López-Gil, M., Angulo Rasco, F. y Vázquez-Recio, R. (2017): Sentido, gravedad y razones del ciberplagio entre el alumnado de ESO de Andalucía, Icono 14, volumen 15 (2), pp. 114-136. doi: 10.7195/ri14.v15i2.1054 


\section{Resumen}

Este artículo tiene como fin mostrar parte de los resultados que se han extraído de la investigación "El plagio académico entre el alumnado de ESO de Andalucía" (EDU2009-14019-C02-02) financiado por el Ministerio de Ciencia e Innovación. El principal objetivo del proyecto fue captar cuál era la concepción del alumnado andaluz de ESO sobre el ciberplagio. Nos centraremos en las prácticas deshonestas en la elaboración de trabajos académicos: su sentido, la gravedad y las razones que las motivan entre las que destacan el desconocimiento ante lo que es plagio, la procastinación y la complejidad de las tareas que se les requieren. Como instrumento de recogida de información se utilizó un cuestionario diseñado por el grupo de investigación Educación y Ciudadanía de la UIB (EiC) (Sureda et al., 2015), del que se obtuvo una muestra de 1.170 alumnos/as. La conclusión más significativa es que la evitación del plagio requiere de un aprendizaje en las tareas de gestión, organización y presentación de información, por una parte, y por otra, la necesidad de formación en los procesos de citación de fuentes.

Palabras clave: Educación secundaria - Bachillerato - Integridad académica Deshonestidad académica - Ciberplagio

\section{Abstract}

This article aims to show some of the lessons and results drawn from the research "The academic plagiarism among students of ESO of Andalusia" with reference EDU2009-14019-C02-02 funded by the Ministry of Science and Innovation. The project aims, among others, was to capture what was the concept that students of ESO in Andalusia entertained on the Cyberplagiarism. The analysis of academic plagiarism is just the tip of the iceberg of a much deeper issue as academic integrity (Morey, Sureda, Oliver \& Comas, 2013; Sureda et al., 2015). We will focus in this article on dishonest practices in the development of academic written-work: their sense, gravity and the reasons that encourage these practices among students in ESO Andalusia. Some reasons are the procasting the ignorance about the plagiarism and the difficulties to do some tasks. It was used as a major research tool a questionnaire designed based on previous research about plagiarism. This questionnaire 
Sentido, gravedad y razones del ciberplagio entre el alumnado de ESO de Andalucía | 116 ARTÍCULOS DE INVESTIGACIÓN

was answered by 1,170 students from Andalusia. The mail conclusion is that the plagiarism requires on the one hand learning about the management, organization and presentation of information and on the other hand knowledge about how to make bibliographic references.

Key Words: Academic plagiarism - Academic integrity - Academic dishonesty Cyber-plagiarism - Secondary education - High school

\section{Introducción}

Pese a los cambios que las tecnologías digitales están propiciando (Tappscot, 1998; Prensky, 2001a, 2001b; Ante \& Holahan, 2008; Schulmaister, 2008), en muchas ocasiones se ha querido centrar el foco en una 'cohorte generacional', en las nuevas generaciones, y ello ha impedido captar la dimensión y profundidad del cambio que se está produciendo en nuestra sociedad y en nuestro modo de convivir, comunicar y entender el conocimiento mismo (Kerckove, 1997; Hutchby \& Moran-Ellis, 2001; Holloway \& Valentine, 2003; Pisani \& Piotet, 2009; Kress, 2010; López \& Angulo, 2015; López, 2017). No obstante, no constituye una cuestión generacional, sino que se trata del imparable surgimiento de una cultura digital (Gere, 2002; Weinberger, 2007; Angulo \& Vázquez, 2010). Si por ejemplo consultamos las últimas estadísticas sobre acceso o uso de Internet en España, podemos darnos cuenta de que, si ya en 2007 el INE informaba que el 51\% de los internautas españoles se encontraba en un intervalo de edad entre 16 y 34 años, actualmente más del 99\% de hombres y el 97\% mujeres (entre 10 y 15 años) habían usado Internet en los últimos meses, cifra que no desciende entre los 25 y 34 años de edad (93,2\% y $94,2 \%$ respectivamente para hombres y mujeres). Podríamos afirmar que en los próximos 10 o 15 años tendremos una mínima población nacida antes del cambio digital en comparación a la que ha nacido, crecido, comunicado y aprendido en dicha cultura.

A nuestro juicio, este marco general es mucho más explicativo, y es en el que tenemos que enmarcar las conductas de deshonestidad académica, especialmente, las conductas de ciberplagio. La nueva cultura digital permite un acceso sencillo y en grandes cantidades de datos y de información no solo escrita, sino también 
audiovisual (Angulo \& Vázquez, 2010; Mut, Morey, Comas \& Negre, 2012). Esta facilidad de acceso es, además, en gran parte ubicua, dada la movilidad intrínseca de las tecnologías digitales, y atemporal, pues nos podemos conectar en cualquier momento. Esta circunstancia hace que el uso de dichos datos y dichas informaciones se convierta en una actuación aceptable y normalizada. Pero esta situación no quita ni resta valor al problema creciente del plagio y ciberplagio académico (McCabe, Treviño \& Butterfield, 2001; Buckley, Wiese \& Harvey, 1998; Ercogevac \& Richardson, 2004; Anderson, Steneck, 2011 \& Mut, 2012; Cheeman, Mahmood, Mahmood \& Shah, 2011), dado que se trata de dos cuestiones relacionadas pero diferentes.

Por ello, nos planteamos la necesidad de profundizar en esta problemática en torno a varias cuestiones de investigación como son:

- ¿Qué concepto de plagio y ciberplagio tiene el alumnado de ESO andaluz?

- ¿Qué conocimiento tiene el alumnado de ESO andaluz en relación a cómo se podría evitar el plagio académico (cómo citar o referenciar, entre otras)?

- ¿Qué razones motivan esta práctica deshonesta?

- ¿Qué valor ético (qué gravedad) le otorga el alumnado al plagio y ciberplagio?

\section{Plagio y ciberplagio: estado de la cuestión}

A pesar de que este artículo centre su atención en la práctica del plagio y ciberplagio, como han señalado Comas y Sureda (2007), el análisis y estudio de este fenómeno es una parte importante de un campo de análisis mucho más extenso denominado integridad académica (Morey et al., 2013; Sureda et al., 2015). La integridad académica engloba, al menos, tres subcampos de estudio que no siempre se analizan por separado, pero que han de ser diferenciados. Según Morey, Sureda, Oliver \& Comas (2014, p. 226), dichos sub-campos son los siguientes: 
Sentido, gravedad y razones del ciberplagio entre el alumnado de ESO de Andalucía | 118

ARTÍCULOS DE INVESTIGACIÓN

a. Prácticas deshonestas en la realización de exámenes o pruebas de evaluación (copia o utilizar 'chuletas').

b. Prácticas deshonestas en la elaboración de trabajos académicos (por ejemplo, utilizar trabajos descargados de alguna plataforma de Internet, como `El Rincón del Vago' en España).

c. Prácticas deshonestas relacionadas con la vida cotidiana de los centros (como dañar material pedagógico o equipamiento de los centros).

Los ámbitos que nos interesan son los dos primeros, pero especialmente el segundo. En este sentido, gran parte de la literatura disponible se ha centrado en analizar la prevalencia, extensión e identidad del plagio académico entendido como deshonestidad en los exámenes y deshonestidad en la elaboración de trabajos. Los estudios de MacCabe, Treviño \& Butterfield (2001), Ercegovac \& Richardson (2004), DeVoss \& Rosati (2002), Heitman \& Litewka (2011), entre otros, son buena muestra de ello. Igualmente, son de interés las investigaciones que se han centrado en la relación entre rendimiento académico y comisión de plagio (Straw, 2002; Finn \& Frone, 2004; Morey et al., 2013), en el análisis de los factores explicativos (Rattinger \& Kramer, 2009) y especialmente en los análisis en el ámbito académico (Volgesang, 1997; Sutherland-Smith, 2005; Blanch, Rey y Folch, 2006; Pupovac, Billic-Zulle \& Petrovecki, 2008; Comas, Sureda, Casero \& Morey, 2011; Heitman \& Ltewka, 2011; Strom, \& Strom, 2007). Destacar también, por su importancia, los estudios que analizan las causas del plagio (Comas, 2009) que han sido agrupadas en: filosófico morales, socio-culturales, pedagógicas, legislativas, tecnológicas y psicológicas. Sin embargo, llama la atención que, en relación a las causas, las personales o individuales no hayan sido tenidas en cuenta, pese a ser enormemente importantes (McCabe \& Treviño, 1997). En relación a esta cuestión estaríamos haciendo referencia a la procastinación (Klassen, Krawchuk \& Rajani, 2008; Sureda, Comas \& Oliver, 2015), pero también en la inmadurez (Haines, Diekhoff, LaBeff \& Clark, 1986). Señalamos este extremo porque en esta investigación hemos podido detectar que justamente las razones personales o individuales son clave en la comisión de ciber-plagio, como veremos posteriormente. En España la literatura sobre el plagio no es tan prolija como cabría esperar. Además de la investigación citada de Blanch, Rey \& Folch (2006), cabe mencionar los pioneros trabajos realizados por el equipo de la Universidad de las Islas Baleares (Comas, 2009; Comas, Sureda, Angulo \& Mut, 2011; Comas, Sureda, Casero \& Morey, 2011; Mut, 2012; Morey et al., 2013; Sureda et al., 2015). 
No obstante, el número de trabajos sobre el plagio académico en los estudios de secundaria y bachillerato es bastante menos numeroso y, de alguna manera, muy novedoso. Destacan el trabajo de Anderman, Griesinger \& Westerfiel (1998) los estudios centrado en factores personales entre jóvenes adolescentes de Murdock, Hale \& Weber (2001), los análisis cualitativos de Ma, Lu \& Wan (2008) en tres escuelas de secundaria; el proyecto GENIUS (Dias \& Bastos, 2014) cuya investigación se centra en 170 docentes y 334 estudiantes de secundaria de Inglaterra, Turkia, Rumanía, Portugal, Italia, Grecia y España; también la investigación sobre el alumnado de bachillerato de Mut (2012), la revisión realizada por Comas, Sureda, Angulo \& Mut (2011) considerada una de las primeras en el campo, el estudio sobre la prevalencia de la comisión del ciberplagio en razón del rendimiento académico con una muestra de 1515 alumnos de 10 centros educativos de Mut, Morey, Comas y Sureda (2012); el estudio centrado en la relación entre plagio y rendimiento académico de Morey, Sureda, Oliver \& Comas (2013) con una muestra de 1503 alumnos de segundo, tercero y cuarto curso de ESO; y el más reciente de Sureda, Comas \& Oliver (2015) que analiza la relación entre el género, la procastrinación y el plagio académico en secundaria. La importancia del estudio del plagio en secundaria no viene justificada solo por sí misma, sino también, y como han indicado Harding, Mayhew, Finelli \& Camperter (2007), las conductas deshonestas en estas edades son un fuerte predictor de la deshonestidad académica en la universidad, lo que a su vez lleva a convertirse en un excelente indicador de las conductas deshonestas en el puesto de trabajo (Harper, 2006). Afirmamos, por tanto, que se hace necesario seguir trabajando en el estudio y el análisis de esta práctica que ha tomado nuevos caminos en la Era Digital. La investigación que presentamos pretende aportar algunas ideas para comprender estas conductas entre el alumnado de secundaria de Andalucía, centrándonos en el sentido, la gravedad y las razones de estas conductas.

\section{Metodología}

El estudio para averiguar el sentido, la gravedad y las razones del ciberplagio entre el alumnado de ESO de Andalucía ha combinado la perspectiva cuantitativa y la cualitativa. En este artículo nos centramos en la fase cuantitativa desarrollada a través de un cuestionario aplicado al alumnado de ESO andaluz cuyos detalles se muestran a continuación. En este apartado detallaremos los aspectos relacionados con la muestra, 
los instrumentos de recogida de información y el proceso de datos. La elección de este instrumento se debe a que nos permite acceder a las ideas y las creencias que una muestra amplia tiene sobre el problema objeto de estudio (Rodríguez Gómez, Gil Flores y García Jiménez, 1996; Martínez Olmo, 2002; Callejo Gallego, 2010).

\subsection{La muestra}

Este estudio se llevó a cabo con una muestra representativa del alumnado de ESO en Andalucía. El muestreo fue aleatorio estratificado, considerando los siguientes estratos: cursos de ESO (de $1^{\circ}$ a $4^{\circ}$ curso) y provincia (Cádiz, Almería, Sevilla, Huelva, Córdoba, Granada y Málaga) constituyendo un total de 1.170 alumnos y alumnas (la edad media de la muestra es de 14,8 años, con $\sigma=1,487$.) de 17 centros públicos y privados/concertados. Cada encuestador, además de un período de formación, contó con un protocolo de aplicación. Ello supone un error muestral de $\pm 2,5 \%$ estimado para un nivel de confianza del $95 \%$ y bajo la condición favorable de $\mathrm{p}=\mathrm{q}=0,5$ (varianza poblacional de 0,25 ). La muestra tiene las características que se detallan en la Tabla $n^{0} 1$.

\begin{tabular}{|c|c|c|}
\hline \multicolumn{2}{|c|}{ CARACTERÍSTICAS } & $\%$ \\
\hline \multirow{2}{*}{ Tipo de Centro } & PÚBLICO & $72,2(902)$ \\
\cline { 2 - 3 } & PRIVADO & $22,8(266)$ \\
\hline \multirow{2}{*}{ Curso } & $1^{\circ}$ ESO & $32,4(378)$ \\
\cline { 2 - 3 } & $2^{\circ}, 3^{\circ}, 4^{\circ}$ & $67,6(790)$ \\
\hline $\begin{array}{c}\text { Género } \\
\text { (Perdidos: 112) }\end{array}$ & MUJER & $53,7(568)$ \\
\cline { 2 - 3 } & HOMBRE & $46,3(490)$ \\
\hline $\begin{array}{c}\text { País de Nacimiento } \\
\text { (Perdidos: } 28 \text { ) }\end{array}$ & ESPAÑA & $95,6(1092)$ \\
\cline { 2 - 3 } & OTROS & $4,4(50)$ \\
\hline
\end{tabular}

Tabla 1: Características de la muestra

Fuente: elaboración propia.

Debemos destacar que en relación al acceso y uso de Internet el 94,5\% del alumnado encuestado dispone de conexión a Internet en casa, frente a un 5,4\% que admite no disponer de este recurso en el hogar. En este marco, el 72,9\% afirma acceder principalmente a Internet en el hogar. 


\subsection{Instrumento de recogida de datos}

El cuestionario ha sido el instrumento para la recogida de los datos. Este, titulado Cuestionario sobre plagio académico entre el alumnado de ESO, ha sido diseñado por el equipo de investigación de la Universidad de las Islas Baleares "Educación y Ciudadanía" (Sureda, Comas y Olivert, 2015) dentro del proyecto de investigación "El plagio académico entre el alumnado de ESO de Andalucía". Dicho cuestionario se elaboró a partir de investigaciones sobre plagio (Comas, 2009; Comas y Sureda, 2010; Sureda, Comas, Morey, Mut \& Gil, 2010; Comas, Sureda, Angulo \& Mut, 2011; Mut, 2012) validado a través de una doble verificación: panel de expertos y aplicación a dos grupos piloto de estudiantes de ESO para analizar la viabilidad y comprensión de la encuesta. El instrumento contempla preguntas dicotómicas, politómicas y de respuesta múltiple. Su estructura se presenta a través de cuestiones pedagógicas (la realización de trabajos), y otras más concretas sobre los objetivos de la investigación (el reconocimiento de lo que sea una referencia bibliográfica, sobre el Rincón del Vago, su percepción de Internet, y preguntas que indagan sobre su sentido de lo que es o no ciber-plagio). Para su empleo en Andalucía se llevaron a cabo mínimas adaptaciones como la supresión de la asignatura de catalán. El cuestionario formado por 14 ítems (de los cuales el número 12 estaba estructurado en torno a 5 viñetas/escenas) fue validado mediante su aplicación previa a una muestra de 75 estudiantes de ESO de las Islas Baleares y la evaluación de 7 expertos en metodología de la investigación social (Mut, Morey, Comas \& Sureda, 2012). El cuestionario se aplicó entre septiembre de 2011 y junio de 2012 de forma individual y anónima en situaciones de aula con la presencia del docente en el horario lectivo.

\subsection{Procesado de Datos}

Hemos realizado un análisis descriptivo, y cuando ha sido posible hemos utilizado estadísticos no paramétricos para encontrar relaciones significativas entre las variables de estudio. Para el conjunto de análisis producidos hemos aplicado el paquete estadístico SPSS (versión 19.0). 


\section{Resultados}

\subsection{El sentido del ciber-plagio}

Uno de los objetivos fundamentales de la investigación consistía en captar cuál era el sentido del ciberplagio, es decir, la concepción que el alumnado de ESO en Andalucía abrigaba sobre este tema, tanto de forma indirecta (por ejemplo, a través de su conocimiento o no de una referencia bibliográfica) como directa a través de la utilización de la plataforma Rincón del Vago o de su respuesta y posicionamiento frente a cuatro situaciones en forma de viñetas que implicaban decisiones sobre utilización/copiado completo de trabajos de la web (viñeta primera), empleo de trabajos elaborados por compañeros (viñeta segunda), copiado parcial de trabajos e información disponible en la red (viñeta cuarta), copiado de información en enciclopedias y libros de consulta (viñeta cuarta) y compra de trabajos. Los resultados no pueden ser más evidentes.

Ante la pregunta de qué era una referencia, a elegir entre 5 posibles opciones, como muestra el Cuadro $n^{0} 1$, solo el $26,4 \%$ del alumnado señaló la respuesta correcta (C), frente a un $38,1 \%$ que se distribuyeron entre las respuestas (A) y (B) y un $35,5 \%$ que afirmó no saber lo que era una referencia (E).

\section{¿Qué es una referencia? Elige una respuesta}

A. La sección inicial de un libro donde se hace un resumen-presentación del contenido

B. El diccionario ideológico de la lengua española

C. Alsina, J. (1983). Literatura griega. Contenidos, métodos y problemas. Barcelona, Editorial Ariel.

D. La sección final de un libro donde se hace un resumen del contenido

E. No sé lo que es una referencia bibliográfica

Cuadro 1: Opciones de respuesta ante la pregunta ¿Qué es una referencia?

Fuente: elaboración propia.

Este claro desconocimiento de lo que es una referencia, elemento clave en relación a la comisión de plagio, se comprende en parte si tenemos en cuenta que el $56,5 \%$ del alumnado afirmó que nunca se le había enseñado qué es una referencia bibliográfica, frente a un $43,5 \%$ que sí. La laguna formativa es un elemento que propicia o puede propiciar la conducta deshonesta. No obstante, queremos 


\section{ARTÍCULOS DE INVESTIGACIÓN}

enfatizar que este hecho no lo explica completamente. También interviene otros factores como el efecto de la procastinación, como han señalado Sureda, Comas \& Oliver (2015) para el alumnado de las Islas Baleares, y otras razones individuales como luego veremos.

Como indicábamos arriba, el cuestionario indaga sobre ese microcosmos de Internet en España, que es el Rincón del Vago. Preguntamos al alumnado sobre el conocimiento de esta web. El 69,2\% conoce dicha plataforma frente a un $30,8 \%$ que afirma desconocerla; conocimiento que deriva esencialmente de su propia indagación en Internet $(38,8 \%)$ y de sus compañeros de colegio $(33,2 \%)$. Sin embargo, cuando se le pregunta si han copiado o utilizado trabajos de dicha plataforma solo el $32,5 \%$ lo afirma, frente a un $67,5 \%$ que lo niega.

Con el propósito de identificar si el alumnado entendía qué era una acción de plagio, el cuestionario presentaba una cita-fragmento tomada de la Wikipedia (identificada correctamente) y tres versiones diferentes de utilización de dicha información. Se les preguntaba que indicasen qué fragmento era copia del original, qué fragmento sería mejor y peor para utilizar en un trabajo.

\begin{tabular}{|c|l|}
\hline \multirow{5}{*}{ Texto original extraído de Wikipedia: } & $\begin{array}{l}\text { La Revolución francesa fue un conflicto social y políti- } \\
\text { co, con diversos periodos de violencia, que convulsionó } \\
\text { Francia y, por extensión de sus implicaciones, a otras } \\
\text { numerosas naciones de Europa que enfrentaban a parti- } \\
\text { darios y opositores del sistema denominado del Antiguo } \\
\text { Régimen. Se inició con la autoproclamación del Tercer } \\
\text { Estado como Asamblea Nacional en } 1789 \text { y finalizó con el } \\
\text { golpe de estado de Napoleón Bonaparte en } 1799 .\end{array}$ \\
\hline Fragmento A & $\begin{array}{l}\text { La Revolución francesa dio comienzo a partir de la au- } \\
\text { toproclamación del Tercer Estado como Asamblea Na- } \\
\text { cional en el año 1789 y concluyó en el año 1799 con el } \\
\text { golpe de estado de Napoleón Bonaparte. Tuvo fuertes } \\
\text { implicaciones en numerosas naciones de Europa y supu- } \\
\text { so el enfrentamiento entre personas a favor y en contra } \\
\text { del Antiguo Régimen. }\end{array}$ \\
\hline
\end{tabular}


Sentido, gravedad y razones del ciberplagio entre el alumnado de ESO de Andalucía | 124

ARTÍCULOS DE INVESTIGACIÓN

\begin{tabular}{|l|l|}
\hline \multirow{2}{*}{ Fragmento B } & La Revolución francesa se inició con la autoproclama- \\
& ción del Tercer Estado como Asamblea Nacional en 1789 \\
y finalizó con el golpe de estado de Napoleón Bonaparte \\
en 1799. Fue un conflicto social y político, con diversos \\
periodos de violencia, que convulsionó Francia y, por ex- \\
tensión de sus implicaciones, a otras numerosas naciones \\
de Europa que enfrentaban a partidarios y opositores del \\
sistema denominado del Antiguo Régimen.
\end{tabular}

Tabla 2: Opciones de respuesta ante la pregunta ¿Qué es plagio?

Fuente: elaboración propia.

A continuación, mostramos la frecuencia con la que se presentan las diferentes escenas y que nos dan cuenta de qué es el plagio para el alumnado (Tabla 3).

\begin{tabular}{|c|c|c|c|c|}
\hline & \% Válido & Plagio & Mejor para entrega de trabajo & Peor para entrega de trabajo \\
\hline \multirow{4}{*}{ Válidos } & Fragmento a & 15,5 & 30,5 & 23,2 \\
\cline { 2 - 6 } & Fragmento b & 27,8 & 41,1 & 15,3 \\
\cline { 2 - 6 } & Fragmento c & 48,8 & 23,2 & 49,7 \\
\cline { 2 - 5 } & No lo sé d & 7,9 & 5,2 & 11,8 \\
\hline
\end{tabular}

Tabla 3: ¿Qué es ciberplagio para el alumnado de ESO?

De los resultados obtenidos podemos deducir cuatro ideas básicas:

1. El alumnado afirma que plagiar es precisamente hacer lo contrario. El $48 \%$ indica que el fragmento $C$ es un plagio, cuando, sensu stricto, es una forma correcta de referenciar una fuente. 


\section{ARTÍCULOS DE INVESTIGACIÓN}

2. Las diferencias en porcentaje entre los fragmentos B $(27.8 \%)$ y C $(48,8 \%)$ se pueden explicar porque una copia más extensa es más plagio que una copia más corta. Dicho de otra manera, copiar más palabras de una fuente es más plagio que copiar menos.

3. Siguiendo la tónica anterior, el fragmento C $(49,7 \%)$ es la peor opción para entregar un trabajo, seguida del fragmento A $(23,2 \%)$, mejor opción en tanto copia sintética.

4. La mejor opción según las respuestas para utilizar en un trabajo es el fragmento A $(30,5 \%)$, seguido del fragmento $B(41,1 \%) \mathrm{y}$, en muy poca medida, el fragmento $\mathrm{C}(23,2 \%)$.

Cuando se pregunta de dónde extraer la información básica a la hora de hacer un trabajo, el 82\% ( $\mathrm{f}=931$ ) afirma que de Internet en casa, Internet en otro lugar (no la escuela) y escasamente utiliza enciclopedias y libros (de bibliotecas o del hogar). Teniendo en cuenta este dato, indagamos sobre qué tipo de plagio se trata. Para ello se le mostraba una serie de viñetas en las que: a) se bajaba información de internet; $b$ ) copiaba un trabajo de un compañero; c) se combinaba información de distintas webs; d) se copiaba la información de libros y enciclopedias; e) se encargaba un trabajo pagando por ello. De los resultados podemos deducir que solo una minoría afirma (entre 1 y más de 5 veces) bajarse trabajos de Internet $(33,4 \%)$, copiar un trabajo de un compañero $(39,3 \%)$ o encargar un trabajo pagado $(18,1 \%)$. Únicamente la escena $C$ en la que se anima a combinar información extraída de páginas web muestra una clara mayoría: $61,1 \%$. En todo caso, el empleo de textos no digitales como fuente de copia alcanza un $42,6 \%$ entre 1 y más de 5 veces (Tabla 4).

\begin{tabular}{|c|c|c|}
\hline ESCENAS & OPCIONES & \% VÁLIDO \\
\hline \multirow{3}{*}{ Escena 1 ${ }^{\mathrm{a}}$} & Nunca & $66,6^{* *}$ \\
\cline { 2 - 3 } & Entre 1 y 5 veces & 29,0 \\
\cline { 2 - 3 } & Más de 5 veces & 4,4 \\
\hline \multirow{3}{*}{ Escena 2a } & Nunca & $60,8^{* *}$ \\
\cline { 2 - 3 } & Entre 1 y 5 veces & 31,6 \\
\cline { 2 - 3 } & Más de 5 veces & 7,7 \\
\hline
\end{tabular}


Sentido, gravedad y razones del ciberplagio entre el alumnado de ESO de Andalucía | 126 ARTÍCULOS DE INVESTIGACIÓN

\begin{tabular}{|c|c|c|}
\hline ESCENAS & OPCIONES & \% VÁLIDO \\
\hline \multirow{3}{*}{ Escena 3 $^{\text {a }}$} & Nunca & 38,3 \\
\cline { 2 - 3 } & Entre 1 y 5 veces & $45,4^{*}$ \\
\cline { 2 - 3 } & Más de 5 veces & 15,7 \\
\hline \multirow{3}{*}{ Escena 4 $^{\mathrm{a}}$} & Nunca & $57,4^{* *}$ \\
\cline { 2 - 3 } & Entre 1 y 5 veces & 33,2 \\
\cline { 2 - 3 } & Más de 5 veces & 9,4 \\
\hline \multirow{3}{*}{ Escena 5 $^{\text {a }}$} & Nunca & $81,9 \%$ \\
\cline { 2 - 3 } & Entre 1 y 5 veces & $11,5 \%$ \\
\cline { 2 - 3 } & Más de 5 veces & $6,6 \%$ \\
\hline
\end{tabular}

Tabla 4: Datos relativos a la cuestión sobre tipología de plagio Fuente: elaboración propia.

Pero ¿qué pasa cuando se le pregunta sobre la conducta de sus compañeros? En este caso las respuestas se giran hacia el lado contrario, como puede apreciarse en la Tabla 5. El alumnado atribuye, en general, a sus compañeros conductas que ellos mismos no se aplican. Manifiestan que son otros los que se bajan información $(55,0 \%)$, copian trabajos de sus compañeros $(59,85)$, copian de libros y enciclopedias $(55,9 \%)$, e incluso encargan trabajos de pago $(84,0 \%)$. Estos resultados no han de interpretarse a la ligera. Una posible explicación es que cuando asignan a otras conductas que ellos no se imputan directamente, están, justamente, mostrándose a sí mismos o, en todo caso, señalando que el ciberplagio está bastante extendido. De todas maneras, solo se atribuyen copiar trozos de información de páginas web (un $21,8 \%$ ).

\begin{tabular}{|c|c|c|}
\hline ESCENAS & OPCIONES & PORCENTAJE \\
\hline \multirow{2}{*}{ Escena 1 $^{\text {a }}$} & Nunca & $45,0 \%$ \\
\cline { 2 - 3 } & Entre 1 y 5 veces $\left(^{*}\right)$ & $55,0 \%$ \\
\hline \multirow{2}{*}{ Escena $2^{\text {a }}$} & Nunca & $40,1 \%$ \\
\cline { 2 - 3 } & Entre 1 y 5 veces & $59,8 \%$ \\
\hline \multirow{2}{*}{ Escena 3 } & Nunca & 28,4 \\
\cline { 2 - 3 } & Entre 1 y 5 veces & 21,8 \\
\hline
\end{tabular}




\section{ARTÍCULOS DE INVESTIGACIÓN}

\begin{tabular}{|c|c|c|}
\hline ESCENAS & OPCIONES & PORCENTAJE \\
\hline \multirow{2}{*}{ Escena 4 ${ }^{\mathrm{a}}$} & Nunca & $44,1 \%$ \\
\cline { 2 - 3 } & Entre 1 y 5 veces & 55,9 \\
\hline \multirow{2}{*}{ Escena 5 $5^{\mathrm{a}}$} & Nunca & 76,1 \\
\cline { 2 - 3 } & Entre 1 y 5 veces & 84,0 \\
\hline
\end{tabular}

Tabla 5: ¿Qué hacen tus compañeros? (Hemos agrupado por comodidad las respuestas entre '1 y 5 veces' y 'más de 5 veces')

Fuente: elaboración propia.

Todo ello nos indica que el alumnado de ESO de Andalucía está muy lejos de entender y diferenciar una cita textual de lo que es plagio, hecho que evidencia que aquí existe un importante déficit en su formación (Anyanwu, 2004).

\subsection{Gravedad del plagio}

Otro elemento que nos interesaba analizar era la idea de 'gravedad' que el alumnado manejaba en relación a diferentes tipos de plagio, algunos de los cuales hemos analizado en el apartado anterior. Para ello solicitamos que se posicionase con respecto a 9 modalidades de copia, según las opciones siguientes: nada grave, poco grave, grave, bastante grave, muy grave y extremadamente grave. No se introdujo una opción intermedia, circunstancia que nos permitía unir los resultados en dos grandes grupos: sin importancia y con importancia, que agrupa respectivamente las tres primeras modalidades y las tres siguientes.

Las razones más graves son las que están relacionadas con aquellas formas de copia que suponen un engaño deliberado en situaciones de evaluación y de examen: copiar con chuleta (75\%), copiar de un compañero $(29,3 \%)$, con un alto índice ambas opciones en la opción 'extremadamente grave'. En segundo lugar, encontramos que 'copiar de material impreso' (libros y enciclopedias), sea en parte o la totalidad del trabajo $(61,8 \%$ y $53,1 \%$ respectivamente), no son consideradas acciones graves, con el añadido de que en dichos casos tiene una alta puntuación en la opción 'nada grave'. En tercer lugar, la copia de los trabajos de compañeros es más grave que copiar los deberes. En cuarto lugar, volvemos a encontrar resultados parecidos a los que señalábamos más arriba en relación a las escenas. Copiar una parte del trabajo de textos y plataformas de internet no solo no es grave ni recha- 
zable (54,7\% 'sin importancia'), sino que copiarlo entero sí lo es $(60,8 \%$, 'con importancia'). En conclusión, lo más grave es la comisión de conductas deshonestas en situaciones de examen o evaluación; lo menos grave, copiar partes del trabajo de Internet o de libros y enciclopedias (Tabla 6).

\begin{tabular}{|c|c|c|c|}
\hline & SIN IMPORTANCIA (\%) & CON IMPORTANCIA & PUNTUACIÓN EXTREMA \\
\hline $\begin{array}{c}\text { Copiar de una chuleta } \\
\text { en un examen }\end{array}$ & 25 & 75 & $\begin{array}{c}\text { 37,4 (Extremadamente } \\
\text { grave) }\end{array}$ \\
\hline $\begin{array}{l}\text { Dejarse copiar por } \\
\text { un compañero en un } \\
\text { examen }\end{array}$ & 39 & 69,3 & $\begin{array}{c}\text { 27,8 (Extremadamente } \\
\text { grave) }\end{array}$ \\
\hline $\begin{array}{l}\text { Copiar de un compa- } \\
\text { ñero de examen }\end{array}$ & 29,1 & 70,9 & $\begin{array}{c}29,7 \text { (Extremadamente } \\
\text { grave) }\end{array}$ \\
\hline $\begin{array}{c}\text { Copiar algunas partes } \\
\text { de internet }\end{array}$ & 54,7 & 45,3 & $\begin{array}{c}\text { 9,2 (Extremadamente } \\
\text { grave) }\end{array}$ \\
\hline $\begin{array}{l}\text { Copiar un trabajo } \\
\text { entero de internet }\end{array}$ & 39,2 & 60,8 & --- \\
\hline $\begin{array}{c}\text { Copiar un trabajo de } \\
\text { un compañero }\end{array}$ & 32,9 & 67,2 & --- \\
\hline $\begin{array}{l}\text { Copiar los deberes de } \\
\text { los compañeros }\end{array}$ & 48,8 & 51,2 & --- \\
\hline $\begin{array}{l}\text { Copiar partes de un } \\
\text { trabajo de libros y } \\
\text { enciclopedias }\end{array}$ & 61,8 & 28,2 & 32,5 (Nada grave) \\
\hline $\begin{array}{l}\text { Copiar un trabajo } \\
\text { entero de libros y } \\
\text { enciclopedias }\end{array}$ & 53,1 & 46,8 & 25,2 (Nada grave) \\
\hline
\end{tabular}

Tabla 6: Gravedad del Plagio

Fuente: elaboración propia.

\subsection{Las razones del plagio}

El cuestionario utilizado planteaba una serie de razones que se podrían agrupar de la siguiente manera:

- Razones sociales: es una conducta generalizada entre compañeros y compañeras. 


\section{ARTÍCULOS DE INVESTIGACIÓN}

- Razones individuales: es una decisión personal en razón de la situación del alumno o de la alumna.

- Razones de índole pedagógica: tiene que ver con la metodología de enseñanza y el proceso de adquisición de conocimientos y el aprendizaje.

- Razones de índole moral: tiene que ver con una decisión basada en lo 'correcto' o 'incorrecto' de la conducta.

\begin{tabular}{|c|c|c|c|}
\hline Razones & Características & $\begin{array}{c}\text { Nada / Poco } \\
\text { importante } \\
(\%)\end{array}$ & $\begin{array}{c}\text { Importante / } \\
\text { Muy importante } \\
(\%)\end{array}$ \\
\hline Porque todo el mundo lo hace & Social & 49,9 & 33,6 \\
\hline Por pereza de hacerlos & \multirow{6}{*}{ Individual } & 24,9 & 47,2 \\
\hline Por falta de tiempo & & 29,9 & 38,3 \\
\hline Por creer que no te van a pillar & & 39,2 & 38,8 \\
\hline Porque es fácil y sencillo & & 24,3 & $50,9\left(^{*}\right)$ \\
\hline Porque no apetece hacerlos & & 33,2 & $45,6\left(^{*}\right)$ \\
\hline $\begin{array}{l}\text { Porque se saca mejor nota que si lo hace } \\
\text { uno mismo }\end{array}$ & & 43,4 & 35,6 \\
\hline Por ser complicados y difíciles de hacer & \multirow{5}{*}{ Pedagógicas } & 32,4 & 39,9 \\
\hline Porque no se entiende bien para qué sirve & & 44,2 & 30,4 \\
\hline Por tener que hacer muchos en el curso & & 40,8 & 23,6 \\
\hline $\begin{array}{l}\text { Por la creencia de que los profesores no los } \\
\text { leen }\end{array}$ & & 41,4 & $37,4\left({ }^{*}\right)$ \\
\hline $\begin{array}{l}\text { Porque ya está escrito y no vale la pena } \\
\text { hacerlo nuevo }\end{array}$ & & 48,3 & $39,6(*)$ \\
\hline Porque no es malo hacerlo & Moral & 36,6 & 25,3 \\
\hline
\end{tabular}

Tabla 7: Razones del plagio (*) Indica que los casos en los que la opción intermedia 'medianamente importante' es $\geq 20 \%$ de las respuestas, reforzando la razón por la que se copia Fuente: elaboración propia.

Teniendo en cuenta los datos obtenidos, podemos afirmar que la explicación social no es precisamente la razón más destacada. De las individuales, la facilidad de copiar (50,9\%), la 'pereza' $(47,2 \%)$ para realizar un trabajo y la 'no apetencia' 
(una forma de procastinación) (45,6\%) destacan por encima de las demás. En cuanto a las pedagógicas, es la 'dificultad y la complejidad' para realizar los trabajos la opción que destaca por encima de las anteriores. Por último, el componente moral no parece ser destacable, lo que podría significar que para el alumnado andaluz el hecho de que no considere el plagio como una práctica académica deshonesta no es una razón para cometerlo.

Una inferencia que podemos extraer es que, dada la dificultad que puede suponer realizar trabajos (búsqueda y organización de información, redacción, etc.) con el añadido de su poca o nula formación para afrontar este tipo de tareas (recordemos que no se trata de 'realizar' resúmenes), hace que la sencillez a la hora de localizar textos y utilizarlos junto con la falta de tiempo sean las razones que, en última instancia, cobren mayor peso en el alumnado.

\section{Conclusiones y discusión}

Una de las más claras conclusiones a las que podemos llegar es que se necesita no solo de una cultura de la honestidad, sino de un mínimo aprendizaje en la gestión, la organización y la presentación de información; desconocer lo que es una referencia o pensar que una cita correcta es un plagio supone crear un campo propicio para la comisión de plagio, aunque sea de manera casi inconsciente. Este es un déficit importante a tener en cuenta. Asimismo, en el alumnado está arraigada la creencia de que el plagio depende de la cantidad, y de que bajarse textos de Internet es mucho menos grave que copiar en una situación de examen. Esta circunstancia posiblemente se debe a que las sanciones y el riesgo sean menos evidentes. Además, la facilidad de acceso y tener la información a la mano, con el añadido de cierto aburrimiento pedagógico en secundaria, no hacen más que amplificar el problema.

Con el ciberplagio nos encontramos ante un problema dilemático sobre el que tenemos la impresión de que no tiene una resolución clara ni taxativa. Es necesario, como indican muchos autores (Wood, 2004; Fisher \& Zigmond, 2011; Heitman \& Litewka, 2011) promover procesos de formación. No obstante, y teniendo en cuenta que bajo el ciberplagio subyace la cultura digital, tendríamos que cambiar la 
mirada y replantearnos cómo gestionar la información y cómo utilizarla. En algún momento tendremos que entenderlo, resituándolo en lo que se ha denominado desde 1996 por el New London Group nuevas alfabetizaciones (Kalantzis \& Cope, 1997; Lankshear \& Knoble, 2003, 2011; Kress, 2007, 2010), o el iText (Howard, 2007), la cultura juvenil que surge de la misma cultura digital (Sefton-Green, 1998; Hutchby \& Moran-Ellis, 2001; Bernal \& Angulo, 2013; Colás, González \& de-Pablos, 2013) el sentido rizomático de identidad individual (Boyd, 2007; López y Angulo, 2015) y la mezcla informacional (Sonvilla-Weiss, 2010). El uso exclusivo de estrategias punitivas o la aplicación de software antiplagio (Ma, Wan \& Lu, 2008) no resuelve el problema. Necesitamos enfoques mucho más creativos, como señalan Dias y Bastos (2013, p. 2531) para poder romper con la dinámica generada en torno a la práctica del plagio dentro de las posibilidades que nos ofrece la alfabetización digital. Al fin y al cabo, las nuevas generaciones son realmente prosumidoras (López \& Angulo, 2015): consumen tanto como producen información. Entendamos sus formas de consumo y alentemos sus capacidades productivas y creativas.

\section{Referencias}

Anderman, E. M., Griesinger, T. \& Westerfield, G. (1998). Motivation and cheating during early adolescence. Journal of Educational Psychology, 90(1), 84-93. doi:10.1037/0022-0663.90.1.84

Anderson, M. S. \& Steneck, N. H. (2011). The Problem of Plagiarism. Urologic Oncology: Seminars and Original Investigations, 29(1), 90-94. doi:10.1016/j. urolonc.2010.09.013

Angulo Rasco, J.F. \& Vázquez Recio, R. (2010). El currículum y los nuevos espacios para aprender. En J. Gimeno Sacristán (Ed.), Saberes e incertidumbres sobre el currículum (pp. 501-526). Madrid: Morata.

Ante, S. E., \& Holahan, C. (2008). Generation MySpace is getting fed up. Business Week, 407, 54-56.

Anyanwu, R. (2004). Lessons on Plagiarism: Issues for Teachers and Learners. International Education Journal, 4(4), 178-186.

Bernal, C. y Angulo, F. (2013). Interacciones de los jóvenes andaluces en las redes sociales. Comunicar, 20(49), 25-30. doi: 10.3916/C40-2013-02-02 
Sentido, gravedad y razones del ciberplagio entre el alumnado de ESO de Andalucía | 132

ARTÍCULOS DE INVESTIGACIÓN

Blanch, C., Rey-Abella, F. \& Folch, A. (2006). Nivel de conducta académica deshonesta entre los estudiantes de una escuela de ciencias de la salud. Enfermería Clínica, 16(2), 57-61. doi:10.1016/S1130-8621(06)71182-2

Boyd, D. (2007). Identity production in a networked culture: Why youth heart MySpace. MédiaMorphoses, 21, 69-80.

Buckley, M. R., Wiese, D. S. \& Harvey, M. G. (1998). An investigation into the dimensions of unethical behaviour. Journal of Education for Business, 73(5), 284- 290.

Callejo Gallego, J. (Coord).(2009). Introducción a las técnicas de investigación social. Madrid: Centro de Estudios Ramón Areces.

Cheeman, Z., Mahmood, S., Mahmood, A., \& Shah, M. (2011). Conceptual awareness of research scholars about plagiarism at higher educational level: Intelectual property right and patent. International Journal of Academic Research, 3(1), 666-671.

Colás-Bravo, P., González-Ramírez, T., \& de Pablos-Pons, J. (2013). Juventud y redes sociales: Motivaciones y usos preferentes. Comunicar, 20(40), 15-23. doi:10.3916/C40-2013-02-01

Comas, R., Morey, M., Mut, B., \& Gili, M. (2010). El ciberplagi acadèmic. Anàlisi del ciberplagi entre l'alumnat de batxillerat de les Illes Balears. Illes Balears: Govern Ills Balears/iBit/OBSI. Recuperado de: https://goo.gl/sbTWTe

Comas, R. \& Sureda, J. (2010). Academic Plagiarism: Explanatory Factors from Students' Perspective. Journal of Academic Ethics, 8(3), 217-232 D0I: 10.1007/ s10805-010-9121-0

Comas, R. (2009). El ciberplagio y otras formas de deshonestidad académica entre el alumnado universitario (Tesis Doctoral inédita), Palma de Mallorca: Universidad de las Islas Baleares.

Comas, R., Sureda, J. Casero, A. \& Morey, M. (2011). La integridad académica entre el alumnado universitario español. Estudios pedagógicos, 37(1), 207-225. doi:10.4067/S0718-07052011000100011

Comas, R., Sureda, J., Angulo, F. \& Mut, T. (2011). Academic Plagiarism amongst Secondary Education Students: State of the Art. 4th International Conference of Education, Research and Innovations Proceedings, 4314-4321. Madrid: IATED.

DeVoss, D. \& Rosati, A. C. (2002). "It wasn't me, was it?" Plagiarism and the Web. Computers and Composition, 19(2), 191-203. doi:10.1016/S87554615(02)00112-3 
Dias, P. C. \& Bastos, A.S. (2013). Plagiarism phenomenon in European countries: results from GENIUS Project. Procedia-Social and Behavioral Science, 116, 25262531. doi:10.1016/j.sbspro.2014.01.605

Ercegovac, Z. \& Richardson, J.V. (2004). Academic Dishonesty, Plagiarism Included, in the Digital Age: A Literature. Review College and Research Libraries, 65(4), 301-318. doi:10.5860/crl.65.4.301

Finn, K. V. \& Frone, M. R. (2004). Academic performance and cheating: Moderating role of school identification and self-efficacy. The Journal of Educational Research, 97(3), 115-121. doi:10.3200/J0ER.97.3.115-121

Fischer, B. A. \& Zigmond, Michael J. (2011). Educational approaches for discouraging plagiarism. Urologic Oncology: Seminars and Original Investigations, 29(1), 100-103 doi: 10.1016/j.urolonc.2010.11.014

Gere, Ch. (2002). Digital culture. London: Reaction Books.

Haines, V., J., Diekhoff, G. M., LaBeff, E. \& Robert, C. (1986). College cheating: Immaturity, lack of commitment, and the neutralizing attitude. Research in Higher Education, 25(4), 342-354. doi:10.1007/BF00992130

Harding, T., Mayhew, M., Finelli, C. \& Carpenter, D. (2007). The Theory of Planned Behavior as a Model of Academic Dishonesty in Engineering and Humanities Undergraduates. Ethics \& Behavior, 17(3), 255-279. doi:10.1080/10508420701519239

Harper, M. (2006). High tech cheating. Nurse Education Today, 26(8), 672-679.

Heitman, E. \& Litewka, S. (2011). International perspectives on plagiarism and considertations for teaching inernational trainees. Urologic Oncology: Seminars and Original Investigations, 29, 104-108.

Holloway, S. L. \& Valentine, G. (2003). Cyberkids. Children in the information age. London: Routledge Falmer.

Howard, R. M. (2007). Understanding "Internet plagiarism". Computers and Composition, 24(1), 3-15.

Hutchby, I. \& Moran-Ellis, J. (2001). Children, technology and culture. The impact of Technologies in children's everyday lives. London: RoutledgeFalmer.

Kalantzis, M., \& Cope, B. (1997). Multiliteracies: rethinking what we mean by literacy and what we teach as literacy the context of global cultural diversity and new communications technologies. Haymarket: Centre for Workplace Communication and Culture. 
Sentido, gravedad y razones del ciberplagio entre el alumnado de ESO de Andalucía | 134 ARTÍCULOS DE INVESTIGACIÓN

Kerckove, D. (1997). Connected intelligences: The arrival of the web society. Toronto: Somerville House.

Klassen, R. M., Krawchuk, L. L. \& Rajani, S. (2008). Academic procrastination of undergraduates: low self-efficacy to self-regulate predicts higher levels of procrastination. Contemporary Educational Psychology, 33(4), 915-931. doi:10.1016/j.cedpsych.2007.07.001

Kress, G. (2007). Literacy in the new media age. London: Routledge.

Kress, G. (2010). The profound shift of digital literacies. En J. Gillen \& D. Barton (2010), Digital literacies. TLR \& London Knowledge Lab. Institute of Education. Recuperado de: https://goo.gl/5YW9Em

Lankshear, C. \& Knobell, M. (2003). New Literacies. Changing knowledge and classroom learning. Buckingham: Open University Press.

Lankshear, C., \& Knobel, M. (2011). New Literacies: Everyday Practices And Social Learning: Everyday Practices and Social Learning. UK: McGraw-Hill Education.

López, M. (2017). Apren-red: internet como dinamizador del aprendizaje. Journal for Educators, Teachers \& Trainers, JETT, 7(2), 142-154. Recuperado de: https:// goo.gl/bz0a77

López, M. y Angulo, J. F. (2015). Sonorona o el rizoma de la cultura digital. Revista Portuguesa de Educação, 28(1), 9-33. doi:10.21814/rpe.7048

Ma, Honguan J., Lu, Eric Y., Turner, S. \& Wan, G. (2006). An empirical investigation of digital cheating and plagiarism among middle school students. American Secondary Education, 35(2), 69-82.

Ma, Honguan J., Wan, G. \& Lu, E.Y. (2008). Digital Cheating and Plagirism in Schools. Theory into Practice, 47, 197-203.

Martínez Olmo, Fr. (2002). El cuestionario: un instrumento para la investigación en las ciencias sociales. Laertes: Barcelona.

McCabe, D. L., \& Trevino, L. K. (1997). Individual and contextual influences on academic dishonesty: A multicampus investigation. Research in higher education, 38(3), 379-396.

McCabe, D.L., Treviño, L. K. \& Butterfield, K. D. (2001). Cheating in Academic Institutions: a Decade of Research. Ethics \& Behavior, 11(3), 219-232. doi:10.1207/S15327019EB1103_2 


\section{ARTÍCULOS DE INVESTIGACIÓN}

Morey, M., Sureda, J.,Oliver, M. y Comas, R. (2013). Plagio y rendimiento académico entre el alumnado de Educación Secundaria Obligatoria. Estudios sobre Educación, 24, 225-244.

Murdock, T., Hale, N. \& Weber, M. J. (2001). Predictors of cheating among Early Adolescents: Academic and Social Motivations. Contemporary Educational Psychology, 26(1), 96-115. doi:10.1006/ceps.2000.1046

Mut, T. (2012). La alfabetización informacional: una aproximación al ciberplagio académico entre el alumnado de bachillerato (Tesis Doctoral inédita). Palma de Mallorca: Universidad de las Islas Baleares.

Mut, T., Morey, M., Comas, R. \& Sureda, J. (2012). Prevalencia en la comisión de ciberplagio entre alumnado de ESO atendiendo a los resultados académicos en asignaturas del área de lengua y literatura. EDUTEC. Revista Electrónica de Tecnología Educativa, 40. Recuperado de: https://goo.gl/0y0TxK

Park, Ch. (2003). In other (People's) words: plagiarism by university studentsliterature and lessons. Assessment \& Evaluation in Higher Education, 28(5), 471-488 doi:10.1080/0260293032000120352

Pisani, F. \& Piotet, D. (2009). La alquimia de las multitudes: Cómo la Web está cambiando el mundo. Barcelona: Paidós.

Prensky, M. R. (2001a). Digital natives, digital immigrants, Part I. On the Horizon 9(5), 1- 6. Recuperado de: https://goo.gl/a0nRW6

Prensky, M. R. (2001b). Digital natives, digital immigrants, Part 2: Do they really think differently? On the Horizon, 9(6), 1-6. Recuperado de: https://goo.gl/ HXS083

Pupovac, V., Bilic-Zulle, L., \& Petrovecki, M. (2008). On academic plagiarism in Europe. An analytical approach based on four studies. Digithum, 10, 13-19. Recuperado de https://goo.gl/TwZ6mL

Rattinger, D.A. \& Kramer, Y. (2009). Situational and personal causes of student cheating. Research in Higher Education, 50, 293-313. doi:10.1007/s11162-0089116-5

Rodríguez Gómez, G., Gil Flores, J. \& García Jiménez, E. (1996). Metodología de la investigación cualitativa. Archidona: Aljibe.

Schulmaister, R. (2008). Gibt es eine "Net Generation". Universität Hamburg. Paper. Zentrum für Hochschul- und Weiterbildung. 
Sentido, gravedad y razones del ciberplagio entre el alumnado de ESO de Andalucía | 136 ARTÍCULOS DE INVESTIGACIÓN

Sefton-Green, J. (1998) (Ed.). Digital diversions: Youth culture in the age of multimedia. London: UCL Press.

Sonvilla-Weiss, S. (Ed.) (2010). Mashup cultures. Wien: Springer-Verlag.

Straw, D. (2002). The plagiarism of generation 'why not?' Community College Week, 14(24), 4-7.

Strom, P. \& Strom, R. (2007). Cheating in Middle School and High School. The Educational Forum, 71(2), 104-116. doi:10.1080/00131720708984924

Sureda, J., Comas, R. \& Oliver, M. (2015). Academic Plagiarism among Secondary and High School Students: Differences in Gender and Procrastination/Plagio académico entre alumnado de secundaria y bachillerato: Diferencias en cuanto al género y la procrastinación. Comunicar, 22(44), 103-110. doi:10.3916/C442015-11

Sutherland-Smith, W. (2005). Pandora's box: Academic perceptions of student plagiarism in writing. Journal of English for Academic Purposes, 4(1), 83-95. doi: 10.1016/j.jeap.2004.07.007

Tapscott, D. (1998). Growing up digital. The rise of the net generation. New York: McGraw-Hill.

Volgesang, J. (1997). Plagiarism- An Act of Stealing. Journal of PeriAnesthesia Nursing, 12(6), 422-425. doi:10.1016/S1089-9472(97)90005-8

Warnken, P. (2004). Managing Technology. The Journal of Academic Librarianship, $30(3), 237-242$.

Weinberger, D. (2007). Everything is miscellaneous. The power of the new digital disorder. New York: Times Books.

Wood, G. (2004). Academic Original Sin: Plagiarism, the Internet, and Librarias. The Journal of Academic Librarianship, 30(3), 237-242. doi:10.1016/j. acalib.2004.02.011

\section{Agradecimientos}

Este artículo muestra parte de los resultados del proyecto “El plagio académico entre el alumnado de ES0 de Andalucía" con referencia EDU200914019-C02-02 financiado por el Ministerio de Ciencia e Innovación. 